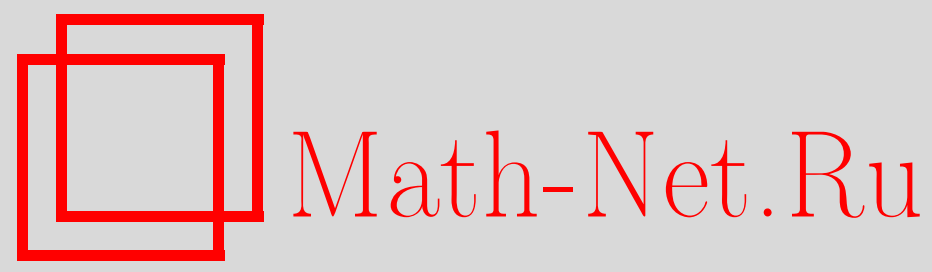

В. А. Краснов, Группа Брауэра неполной вещественной алгебраической поверхности, Матем. заметки, 2000, том 67, выпуск 3, 355-359

DOI: https://doi.org/10.4213/mzm848

Использование Общероссийского математического портала Math-Net.Ru подразумевает, что вы прочитали и согласны с пользовательским соглашением http://www . mathnet.ru/rus/agreement

Параметры загрузки:

IP: 54.198 .67 .100

26 апреля 2023 г., $12: 21: 21$

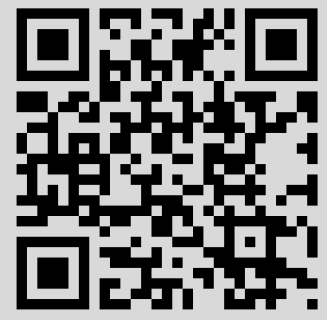




\section{ГРУППА БРАУЭРА НЕПОЛНОЙ ВЕЩЕСТВЕННОЙ АЛГЕБРАИЧЕСКОЙ ПОВЕРХНОСТИ}

\section{В. А. Краснов}

Вычисляется группа Брауэра неполной вещественной алгебраической поверхности. Вьчисления проводятся с помощью эквивариантных когомологий. В результате получается формула, аналогичная формуле для полной поверхности, но доказательство ее имеет существенные отличия.

Библиография: 7 названий.

Введение. Далее $X$ - неособая вещественная квазипроективная алгебраическая поверхность; $\operatorname{Br} X$ - ее группа Брауэра. Если $X$ проективная поверхность, то в работе [1] доказано существование изоморфизма

$$
\operatorname{Br} X=(\mathbb{Q} / \mathbb{Z})^{\rho_{0}(X)} \oplus H^{3}\left(X(\mathbb{C}) ; G, \mathbb{Z}_{-}\right)_{\text {tors }},
$$

где $\rho_{0}(X)$ - число Лефшеца, оно равно рангу трансцендентной групшы когомологий

$$
H_{\text {tr }}^{2}\left(X(\mathbb{C}) ; G, \mathbb{Z}_{-}\right)=H^{2}\left(X(\mathbb{C}) ; G, \mathbb{Z}_{-}\right) / H_{\text {alg }}^{2}\left(X(\mathbb{C}) ; G, \mathbb{Z}_{-}\right) .
$$

Заметим, что $G=G(\mathbb{C} / \mathbb{R})$ - группа Галуа; $\mathbb{Z}_{-}-G$-модуль целых чисел, на котором инволюция комплексного сопряжения $g \in G$ действует умножением на $-1 ; H^{q}(X(\mathbb{C}) ; G$, $\left.\mathbb{Z}_{-}\right)$- группа эквивариантных когомологий, $H^{q}\left(X(\mathbb{C}) ; G, \mathbb{Z}_{-}\right)_{\text {tors }}$ - ее подгрупша кручения; $H_{\mathrm{alg}}^{2}\left(X(\mathbb{C}) ; G, \mathbb{Z}_{-}\right)$- алгебраическая группа когомологий, она равна образу отображения цикла

$$
\text { cl: } \operatorname{Div} X \rightarrow H^{2}\left(X(\mathbb{C}) ; G, \mathbb{Z}_{-}\right) .
$$

В этой заметкемы показываем, что равенство (1) имеет место и для неполной поверхности. Нужно отметить, что доказательство в [1] для неполной поверхности не проходит, так как применяемые там трансцендентные методы годятся только для проективной поверхности. Здесь мы применяем методы и результаты из работы [2].

1. Доказательство основного результата. Рассмотрим этальную топологию $X_{\text {et }}$ на $X$ и пучок $\mathbb{G}_{m}$ на $X_{\text {et }}$, определенньй равенством $\mathbb{G}_{m}(U)=\Gamma(U, \mathscr{O})^{*}$. Тогда существует канонический изоморфизм (см. [3])

$$
\operatorname{Br} X=H_{\mathrm{et}}^{2}\left(X, \mathbb{G}_{m}\right) .
$$

Пусть $\mu_{\infty}(U)$ - группа всех корней из 1 в $\Gamma(U, \mathscr{O})$; тогда получим предпучок $\mu_{\infty}$ на $X_{\text {et }}$; он является пучком, что вытекает из квазикомпактности $X$. Так как $\mu_{\infty}-$ подпучок $\mathbb{G}_{m}$, 
определен фактор-пучок $\mathbb{G}_{m} / \mu_{\infty}$, которьй обозначим через $\varphi$. В работе [2] показано, что гомоморфизм возведения в степень

$$
()^{n}: \varphi \rightarrow \varphi
$$

является изоморфизмом при каждом $n \in \mathbb{N}$. Это означает, что пучок абелевых групп $\varphi$ является пучком векторных пространств над $\mathbb{Q}$ (если групповую операцию в $\varphi$ записывать аддитивно). Из короткой точной последовательности пучков на $X_{\text {et }}$

$$
1 \rightarrow \mu_{\infty} \rightarrow \mathbb{G}_{m} \rightarrow \varphi \rightarrow 1
$$

получаем длинную точную последовательность когомологий

$$
\cdots \rightarrow H_{\mathrm{et}}^{1}(X, \varphi) \stackrel{\delta}{\rightarrow} H_{\mathrm{et}}^{2}\left(X, \mu_{\infty}\right) \rightarrow H_{\mathrm{et}}^{2}\left(X, \mathbb{G}_{m}\right) \rightarrow H_{\mathrm{et}}^{2}(X, \varphi) \stackrel{\delta}{\rightarrow} \cdots
$$

Так как $H_{\mathrm{et}}^{2}\left(X, \mathbb{G}_{m}\right)$ - группа кручения (см. [3]), а $H_{\mathrm{et}}^{2}(X, \varphi)$ - векторное пространство над $\mathbb{Q}$, гомоморфизм

$$
H_{\mathrm{et}}^{2}\left(X, \mathbb{G}_{m}\right) \rightarrow H_{\mathrm{et}}^{2}(X, \varphi)
$$

нулевой. Следовательно, из точной последовательности (3) вытекает равенство

$$
H_{\mathrm{et}}^{2}\left(X, \mathbb{G}_{m}\right)=H_{\mathrm{et}}^{2}\left(X, \mu_{\infty}\right) / \delta\left(H_{\mathrm{et}}^{1}(X, \varphi)\right) .
$$

В работе [2] показано, что существует (неканонический) изоморфизм

$$
H_{\mathrm{et}}^{2}\left(X, \mu_{\infty}\right)=(\mathbb{Q} / \mathbb{Z})^{\alpha} \oplus H^{3}\left(X(\mathbb{C}) ; G, \mathbb{Z}_{-}\right)_{\text {tors }},
$$

где $\alpha$ равно размерности пространства $H^{2}\left(X(\mathbb{C}), \mathbb{Q}_{-}\right)^{G}$, a $\mathbb{Q}_{-}-G$-модуль, равный $\mathbb{Q} \otimes \mathbb{Z}$ _. Отметим, что в [2] рассматриваются полные многообразия, но доказательство равенства (5), предложенное там, проходит и для неполных многообразий. Так как $H_{\text {et }}^{1}(X, \varphi)$ - векторное пространство над $\mathbb{Q}$, из равенства (5) вытекает, что группа $\delta\left(H_{\mathrm{et}}^{1}(X, \varphi)\right)$ содержится в делимой подгруппе $(\mathbb{Q} / \mathbb{Z})^{\alpha}$, а также, что фактор-группа $(\mathbb{Q} / \mathbb{Z})^{\alpha} / \delta\left(H_{\text {et }}^{1}(X, \varphi)\right)$ равна групше вида $(\mathbb{Q} / \mathbb{Z})^{\beta}$. Таким образом, из равенств $(2)$, (4), (5) вытекает равенство

$$
\operatorname{Br} X=(\mathbb{Q} / \mathbb{Z})^{\beta} \oplus H^{3}\left(X(\mathbb{C}) ; G, \mathbb{Z}_{-}\right)_{\text {tors }},
$$

и нам остается доказать, что $\beta=\rho_{0}(X)$.

Пусть $p$ - простое число такое, что $p$-периодическая подгрупша ${ }_{p} H^{3}\left(X(\mathbb{C}) ; G, \mathbb{Z}_{-}\right)$ группы $H^{3}\left(X(\mathbb{C}) ; G, \mathbb{Z}_{-}\right)$равна нулю. Тогда из равенства $(6)$ следует, что достаточно установить для одного из таких $p$ равенство

$$
\operatorname{dim}_{p} \operatorname{Br} X=\rho_{0}(X),
$$

где ${ }_{p} \operatorname{Br} X-p$-периодическая подгруппа $\operatorname{Br} X$.

Для этого рассмотрим точную последовательность Куммера на $X_{\text {et }}$ (см. [4])

$$
1 \rightarrow \mu_{p} \rightarrow \mathbb{G}_{m} \stackrel{()^{p}}{\rightarrow} \mathbb{G}_{m} \rightarrow 1
$$


Из соответствующей длинной точной последовательности когомологий получаем равенства

$$
\operatorname{dim}_{p} \operatorname{Br} X=\operatorname{dim}_{p} H_{\mathrm{et}}^{2}\left(X, \mathbb{G}_{m}\right)=\operatorname{dim} H_{\mathrm{et}}^{2}\left(X, \mu_{p}\right) / \delta_{p}\left(H_{\mathrm{et}}^{1}\left(X, \mathbb{G}_{m}\right)\right),
$$

где $\delta_{p}: H_{\mathrm{et}}^{1}\left(X, \mathbb{G}_{m}\right) \rightarrow H_{\mathrm{et}}^{2}\left(X, \mu_{p}\right)$ - кограничный гомоморфизм. Групша $H_{\mathrm{et}}^{2}\left(X, \mu_{p}\right)$ равна групше $H^{2}\left(X,(\mathbb{C}) ; G, \mu_{p}\right)$, где $\mu_{p}-G$-модуль комплексных корней $p$-й степени из 1 (см. [1], [5]). Далее мы покажем, что гомоморфизм

$$
\begin{array}{cc}
\delta_{p}: H_{\mathrm{et}}^{1}\left(X, \mathbb{G}_{m}\right) & H_{\mathrm{et}}^{2}\left(X, \mu_{p}\right) \\
\| & \| \\
\operatorname{Pic} X & H^{2}\left(X,(\mathbb{C}) ; G, \mu_{p}\right)
\end{array}
$$

равен композиции гомоморфизмов

$$
\begin{aligned}
& \operatorname{Pic} X \stackrel{\mathrm{cl}}{\longrightarrow} H^{2}\left(X(\mathbb{C}) ; G, \mathbb{Z}_{-}\right) \stackrel{R_{p}}{\longrightarrow} H^{2}\left(X(\mathbb{C}) ; G, \mathbb{Z}_{-} / p\right) \\
& \| \\
& H^{2}\left(X,(\mathbb{C}) ; G, \mu_{p}\right) \text {, }
\end{aligned}
$$

где $R_{p}$ - гомоморфизм редукции. Прежде чем доказывать это общее утверждение, покажем, что из него следует равенство (7) при условии, что групшы $H^{3}\left(X(\mathbb{C}) ; G, \mathbb{Z}_{-}\right)$, ${ }_{p} H_{\mathrm{tr}}^{2}\left(X(\mathbb{C}) ; G, \mathbb{Z}_{-}\right)$нулевые.

Действительно, из равенства нулю групшы $H^{3}(X(\mathbb{C}) ; G, \mathbb{Z}-)$ следует, что гомоморфизм редукции

$$
R_{p}: H^{2}\left(X(\mathbb{C}) ; G, \mathbb{Z}_{-}\right) \rightarrow H^{2}\left(X(\mathbb{C}) ; G, \mathbb{Z}_{-} / p\right)
$$

эпиморфен. Следовательно, он индуцирует эпиморфизм

$$
\begin{aligned}
H_{\mathrm{tr}}^{2}\left(X(\mathbb{C}) ; G, \mathbb{Z}_{-}\right) & =H^{2}\left(X(\mathbb{C}) ; G, \mathbb{Z}_{-}\right) / H_{\mathrm{alg}}^{2}\left(X(\mathbb{C}) ; G, \mathbb{Z}_{-}\right) \\
& \rightarrow H_{\mathrm{et}}^{2}\left(X, \mu_{p}\right) / \delta_{p}(\operatorname{Pic} X) .
\end{aligned}
$$

Заметим, что этот эпиморфизм определен в силу утверждения о совпадении гомоморфизма (8) с композицией (9). Так как гомоморфизм

$$
H^{2}\left(X(\mathbb{C}) ; G, \mathbb{Z}_{-}\right) / p \rightarrow H^{2}\left(X(\mathbb{C}) ; G, \mathbb{Z}_{-} / p\right)
$$

мономорфен, то гомоморфизм

$$
H_{\mathrm{tr}}^{2}\left(X(\mathbb{C}) ; G, \mathbb{Z}_{-}\right) / p \rightarrow H_{\mathrm{et}}^{2}\left(X, \mu_{\infty}\right) / \delta_{p}(\operatorname{Pic} X)
$$

индуцированньй гомоморфизмом (10), является изоморфизмом. Таким образом, имеем равенство

$$
\operatorname{dim}_{p} \operatorname{Br} X=\operatorname{dim} H_{\mathrm{tr}}^{2}\left(X(\mathbb{C}) ; G, \mathbb{Z}_{-}\right) / p=\rho_{0}(X),
$$

т.е. вьполняется равенство (7). Перейдем к доказательству того, что гомоморфизм (8) равен композиции гомоморфизмов (9). 
Пусть $\mathscr{A}$ - пучок ростков непрерывных комплекснозначньх функций на $X(\mathbb{C}), \mathscr{A}^{*}-$ пучок обратимых функций. Рассмотрим точную последовательность Куммера $G$-пучков на $X(\mathbb{C})$

$$
1 \rightarrow \mu_{p} \rightarrow \mathscr{A}^{*} \stackrel{()^{p}}{\rightarrow} \mathscr{A}^{*} \rightarrow 1 .
$$

Из нее получаем кограничньй гомоморфизм

$$
\delta_{p}: H^{1}\left(X(\mathbb{C}) ; G, \mathscr{A}^{*}\right) \rightarrow H^{2}\left(X(\mathbb{C}) ; G, \mu_{p}\right) .
$$

Рассмотрим также экспоненциальную точную последовательность $G$-пучков на $X(\mathbb{C})$

$$
0 \rightarrow \mathbb{Z}_{-} \stackrel{2 \pi i}{\longrightarrow} \mathscr{A} \stackrel{\exp }{\longrightarrow} \mathscr{A}^{*} \rightarrow 1 .
$$

Из нее получаем кограничньй гомоморфизм

$$
\delta: H^{1}\left(X(\mathbb{C}) ; G, \mathscr{A}^{*}\right) \rightarrow H^{2}\left(X(\mathbb{C}) ; G, \mathbb{Z}_{-}\right) .
$$

Определен канонический гомоморфизм

$$
\operatorname{Pic} X \rightarrow H^{1}\left(X(\mathbb{C}) ; G, \mathscr{A}^{*}\right),
$$

композиция которого с гомоморфизмом (12) равна отображению цикла (см. [6]):

$$
\text { cl: } \operatorname{Pic} X \rightarrow H^{2}\left(X(\mathbb{C}) ; G, \mathbb{Z}_{-}\right) \text {. }
$$

Рассмотрим коммутативную диаграмму из $G$-пучков на $X(\mathbb{C})$

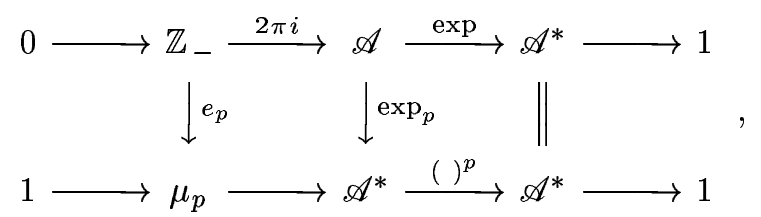

где $e_{p}(n)=e^{2 \pi n i / p}, \exp _{p}(f)=\exp (f / p)$. Из левого столбца диаграммы (13) получаем гомоморфизм редукции по $\bmod p$ :

$$
R_{p}: H^{2}\left(X(\mathbb{C}) ; G, \mathbb{Z}_{-}\right) \rightarrow H^{2}\left(X(\mathbb{C}) ; G, \mu_{p}\right) .
$$

Поэтому из диаграммы (13) следует, что композиция гомоморфизма (12) с гомоморфизмом редукции дает кограничный гомоморфизм (11). Рассмотрим теперь следующую коммутативную диаграмму

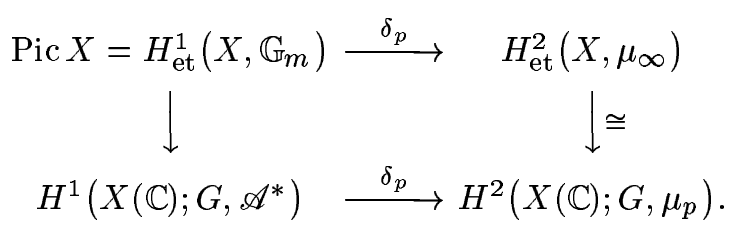

Из нее мы получаем, что гомоморфизм (8) совпадает с композицией гомоморфизмов

$$
\operatorname{Pic} X \rightarrow H^{1}\left(X(\mathbb{C}) ; G, \mathscr{A}^{*}\right) \stackrel{\delta_{p}}{\longrightarrow} H^{2}\left(X(\mathbb{C}) ; G, \mu_{p}\right)
$$

Выше установлено, что гомоморфизм $\delta_{p}$ в ней равен композиции гомоморфизмов

$$
H^{1}\left(X(\mathbb{C}) ; G, \mathscr{A}^{*}\right) \stackrel{\delta}{\rightarrow} H^{2}\left(X(\mathbb{C}) ; G, \mathbb{Z}_{-}\right) \stackrel{R_{p}}{\rightarrow} H^{2}\left(X(\mathbb{C}) ; G, \mu_{p}\right) .
$$

Если дополнить эту строку слева гомоморфизмом

$$
\operatorname{Pic} X \rightarrow H^{1}\left(X(\mathbb{C}) ; G, \mathscr{A}^{*}\right),
$$

то получим композицию $R_{p}$ ○ $\mathrm{cl}$, т.е. гомоморфизм (9), а с другой стороны получаем разложение гомоморфизма (8). Итак, гомоморфизм (8) совпадает с композицией (9). 
ЗАмечАниЕ 1 . На самом делегруппа $H_{\mathrm{tr}}^{2}\left(X(\mathbb{C}) ; G, \mathbb{Z}_{-}\right)$свободная, т.е. равна $\mathbb{Z}^{\rho_{0}(X)}$. Действительно, из выше приведенного доказательства вытекает, что для любого простого числа $p$ вьполняется равенство

$$
\operatorname{dim}_{p} \operatorname{Br} X=\rho_{0}(X)+\operatorname{dim}_{p} H_{\mathrm{tr}}^{2}\left(X(\mathbb{C}) ; G, \mathbb{Z}_{-}\right)+\operatorname{dim}_{p} H^{3}\left(X(\mathbb{C}) ; G, \mathbb{Z}_{-}\right)
$$

С другой стороны, из разложения (1) имеем равенство

$$
\operatorname{dim}_{p} \operatorname{Br} X=\rho_{0}(X)+\operatorname{dim}_{p} H^{3}\left(X(\mathbb{C}) ; G, \mathbb{Z}_{-}\right)
$$

ЗАМЕЧАНИЕ 2. Если $p \neq 2$, то (см. [1])

$$
{ }_{p} H^{3}\left(X(\mathbb{C}) ; G, \mathbb{Z}_{-}\right)={ }_{p} H^{3}\left(X(\mathbb{C}), \mathbb{Z}_{-}\right)^{G},
$$

а поэтому при $p \neq 2$ выполняется равенство

$$
\operatorname{dim}_{p} \operatorname{Br} X=\rho_{0}(X)+\operatorname{dim}_{p} H^{3}\left(X(\mathbb{C}), \mathbb{Z}_{-}\right)^{G} .
$$

В заметке [7] приведена формула для вычисления размерности 2 $\operatorname{Br} X$ для полной поверхности, не использующая эквивариантных когомологий. Рассуждения, проведенные в [7], можно применить и для неполной поверхности. Тогда получается равенство

$$
\operatorname{dim}_{2} \operatorname{Br} X=\rho_{0}(X)-b_{-}^{3}(X)+k^{1}(Y)+s+s_{c}
$$

где $b_{-}^{3}(X)=\operatorname{dim} H^{3}\left(X(\mathbb{C}), \mathbb{Q}_{-}\right)^{G}, Y=X(\mathbb{C}) / G, k^{1}(Y)$ - размерность ядра гомоморфизма ограничения

$$
H^{1}\left(Y, \mathbb{F}_{2}\right) \rightarrow H^{1}\left(X(\mathbb{R}), \mathbb{F}_{2}\right),
$$

$s$ - число компонент связности множества $X(\mathbb{R}), s_{c}$ - число компактных компонент связности множества $X(\mathbb{R})$.

\section{СПИСОК ЦИТИРОВАННОЙ ЛИТЕРАТУРЫ}

[1] Краснов В. А. Когомологическая группа Брауэра вещественного алгебраического многообразия // Изв. РАН. Сер. матем. 1996. Т. 60. № 5. С. 57-88.

[2] Краснов В.А. Аналоги неравенства Гарнака-Тома для вещественной алгебраической поверхности // Изв. РАН. Сер. матем.. (в печати).

[3] Grothendick A. Le groupe de Brauer // Dix exposés sur la cohomologie des schemas. Amsterdam: North-Holland, 1968. P. 46-188.

[4] Милн Дж. Этальные когомологии. М.: Мир, 1983.

[5] Nikulin V. V. On the Brauer group of real algebraic surfaces // Algebraic Geometry and Its Applications. Yaroslavl', 1992. C. 114-136.

[6] Краснов В.А. Характеристические классы векторных расслоений на вещественном алгебраическом многообразии // Изв. АН СССР. Сер. матем. 1991. Т. 55. № 4. С. 716-746.

[7] Краснов В.А. О группе Брауэра вещественной алгебраической поверхности // Матем. заметки. 1996. Т. 60. №6. С. 935-938. 